\title{
Ex vivo cartilage explant model for the evaluation of chondrocyte-targeted exosomes
}

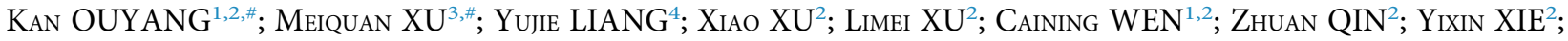 \\ HuAWEI ZHANG ${ }^{5}$; Li DUAN ${ }^{2, *}$; DAPING WANG ${ }^{1,2,5, *}$ \\ ${ }^{1}$ Guangzhou Medical University, Guangzhou, 510095, China \\ 2 Department of Sports Medicine, Guangdong Provincial Research Center for Artificial Intelligence and Digital Orthopedic Technology, The First Affiliated \\ Hospital of Shenzhen University, Shenzhen Second People's Hospital, Shenzhen, 518035, China \\ ${ }^{3}$ Deparment of Pathology, The First Affiliated Hospital of Shenzhen University, Shenzhen Second People's Hospital, Shenzhen, 518035, China \\ ${ }^{4}$ Shenzhen Key Laboratory for Psychological Healthcare, Shenzhen Institute of Mental Health, Shenzhen Kangning Hospital, Shenzhen Mental Health Center, \\ Shenzhen, 518020, China \\ ${ }^{5}$ Department of Biomedical Engineering, Southern University of Science and Technology, Shenzhen, 518055, China
}

Key words: Cartilage explants, Exosomes, Targeted delivery

\begin{abstract}
There is no efficient tracking system available for the therapeutic molecules delivered to cartilage. The dense matrix covering the cartilage surface is the main biological barrier that the therapeutic molecules must overcome. In this study, we aimed to establish a system that can dynamically and effectively track the therapeutic molecules delivered to cartilage. To this aim, we adopted bovine and human cartilage explants as ex vivo models for chondrocyte-targeted exosome dispersion. The efficiency of drug delivery was evaluated using frozen sections. The results of this study showed that the penetration and distribution of chondrocyte-targeted exosomes in cartilage explants can be tracked dynamically. Thus, ex vivo cartilage explants provide an effective and economic system to evaluate therapeutic drugs encapsulated in chondrocyte-targeted exosomes in preclinical studies.
\end{abstract}

\section{Introduction}

Osteoarthritis (OA) is a mainly cartilage-degenerated joint disease that has become a severe threat to public health and a large financial expenditure burden. No effective strategy is available to stop cartilage degeneration (Abramoff and Caldera, 2020). One of the challenges of nonsurgical treatment is that it barely stops OA inflammation microenvironment-induced cartilage matrix degradation (Liang et al., 2021b). Therefore, the development of a targeted system that delivers therapeutics to cartilage defects is urgently needed (Maudens et al., 2018). Another obstacle in strategy development is the lack of an ideal tracking and evaluation system. OA animal models are often used in preclinical studies to evaluate the treatment strategy outcomes, which is a time-consuming process and cannot accurately reflect the OA progression of patients (Nganvongpanit et al., 2008; Nganvongpanit et al., 2009). In

\footnotetext{
*Address correspondence to: Li Duan, duanl@szu.edu.cn; Daping Wang, wangdp@mail.sustech.edu.cn

${ }^{\#}$ These authors contributed equally to this work

Received: 18 August 2021; Accepted: 26 September 2021
}

the clinic, cartilage degeneration can only be examined using magnetic resonance imaging (MRI) scans, which are expensive and require special equipment (Nganvongpanit et al., 2009; Wei et al., 2015). Furthermore, the nature of regenerated cartilage can not be easily diagnosed even by a highly sensitive noninvasive method MRI. Strategies for diagnosing the nature of cartilage are expected to be developed.

Recent studies have demonstrated that cartilage defects are often repaired with fibrous cartilage mainly composed of type I collagen (Nganvongpanit et al., 2009). However, hyaline cartilage is a unique tissue that can permanently perform and maintain joint function, which is the ultimate goal that cartilage regeneration studies aim to achieve (Armiento et al., 2019). Diagnosing the nature of the regenerated cartilage in defects first requires arthroscopic biopsies and then histological immunochemistry assays with type II collagen, a typical hyaline cartilage marker. Orthopedic surgeons seldom perform an arthroscopic biopsy to diagnose the regenerated cartilage after treatment due to ethical concerns and patient willingness. More importantly, arthroscopic biopsy results indicate the outcome of therapy instead of monitoring the treatment progression in realtime. Strategies that can track treatment molecules will contribute to therapeutic development and improvement. 
Extensive studies have been carried out to develop cartilage-targeted drug delivery systems, showing strong potential for hyaline cartilage regeneration in OA treatment (Kou et al., 2019; Liang et al., 2021a). Our previous studies established a cartilage-targeted drug delivery system by designing the chondrocyte affinity peptide (CAP) (Liang et al., 2020). However, a quick and accurate approach to monitoring drug delivery and evaluate the treatment outcome is urgently needed. To this aim, this study established ex vivo cartilage explant models to prove that targeted exosomes can promote the delivery of miR-140 to chondrocytes compare to the native exosomes.

\section{Materials and Methods}

\section{Production of CAP-exosomes}

The transfection of dendritic cells with the CAP-GFP-lamp2b plasmid and plasmid constructions were performed according to Duan L and Liang Y. Dendritic cells in a 12-well plate were transfected in a sterile incubator at $37^{\circ} \mathrm{C}$ and $5 \% \mathrm{CO}_{2}$. Stable cell lines were seeded at a density of $5 \times 10^{6}$ in a $15-\mathrm{cm}$ dish. The cells were cultured in Eagle's essential medium containing $10 \%$ exosome-free FBS. The cell culture supernatants were harvested after $48 \mathrm{~h}$.

The cell culture supernatants were centrifuged at $4^{\circ} \mathrm{C}$ for $10 \mathrm{~min}$ at $300 \times g$ to remove larger particles. Second, the supernatants were further centrifuged at $4^{\circ} \mathrm{C}$ for $15 \mathrm{~min}$ at $2000 \times g$ to remove cell debris. Then, the supernatants were filtered using a $0.22-\mu \mathrm{m}$ filter (Merck Millipore, Burlington). The filtrate was centrifuged at $4^{\circ} \mathrm{C}$ for $30 \mathrm{~min}$ at $10000 \times g$ to remove larger vesicles. Finally, the supernatants were centrifuged at $4^{\circ} \mathrm{C}$ for $70 \mathrm{~min}$ at $120,000 \times g$. The exosome pellets were resuspended in $500 \mu \mathrm{L} 1 \times \mathrm{PBS}$.

microRNA-140 (miR-140) were loaded into exosomes by an electroporation complete system (BIO-RAD, GENE, America). Exosomes were incubated with cy3-miR-140 for $20 \mathrm{~min}$. The voltage of the electrotransmitter was set to $250 \mathrm{~V}$, and the capacitance was set to $125 \mu \mathrm{F}$ (El-Andaloussi et al., 2012). Exosomes were diluted to $0.5-1 \mathrm{mg} / \mathrm{mL}$ with $2 \times$ electroporation solution, and the final volume was $500 \mu \mathrm{L}$. miR-140 were transfected into exosomes using power shock 2-3 times and then incubated for $30 \mathrm{~min}$. Then, the empty carriers were removed using Amicon Ultra 0.5 Centrifugal Filter Unit $100 \mathrm{kDa}$. The centrifugal filter unit was centrifuged at $5000 \mathrm{rpm}$ for $5 \mathrm{~min}$, and washed twice with PBS. Finally, exosomes were resuspended in $200 \mu \mathrm{L} 1 \times$ PBS.

\section{Characterization of exosomes}

The exosome marker proteins in this study included CD9 (1:1000 dilution, ABclonal, A19027), CD81 (1:1000 dilution, ABclonal, A5270), Flotillin (1:1000 dilution, ABclonal, A3023) and Calnexin (1:1000 dilution, ABclonal, No. A15631). The protein expression level was tested by western blotting. For size distribution analysis, exosomes were diluted to $500 \mathrm{ng} / \mathrm{mL}$ and the size distribution was determined with fast video capture and particle-tracking software on a NanoSight NS300 (Malvern Panalytical, UK). For morphology analysis, exosomes were dripped onto formvar carbon-coated nickel grids, negatively stained with $2 \%$ uranyl acetate for $10 \mathrm{~min}$, and visualized by transmission electron microscope (TEM) (JEOL Ltd., JEM2000EX TEM, Tokyo, Japan).

\section{Culture of the ex vivo cartilage}

Human articular cartilage was obtained from femoral heads of 3 patients undergoing total hip joint replacement. This study has been approved by the ethics committees from Shenzhen Second People's Hospital (20201109001-FS01). Cartilage explants were harvested within $2 \mathrm{~h}$ of clinical surgery (Kleuskens et al., 2021; Wuelling and Vortkamp, 2014). Bovine cartilage tissue explants were obtained from three mature bovine femoropatellar grooves immediately after slaughter in the meat-producing plant (Fig. 1). Joint cartilage was excluded from the study, which included visibly roughened surfaces where the superficial layer had lost its smooth architecture and had filled with fibrous cartilage (Kleuskens et al., 2021). The full-thickness harvested cartilage explants were using a 6-mm dermal punch $(6 \mathrm{~mm}$ in diameter and $2 \mathrm{~mm}$ in thickness), then washed ex vivo with PBS three times and incubated with cell culture medium (DMEM, $100 \mu \mathrm{g} / \mathrm{mL}$ streptomycin and $100 \mathrm{U} / \mathrm{mL}$ penicillin, $40 \mu \mathrm{g} / \mathrm{mL} \mathrm{L}$-proline) in 24-well plate.

\section{Exosome penetration in the cartilage}

Cy3-labeled miR-140 was loaded into CAP-exosomes by electroporation. CAP-exosome/miR-140 was added to a 24-well plate and cocultured with human/bovine cartilage explants. The culture medium contained 10\% exosome-free FBS and $1 \%$ penicillin-streptomycin. The coculture system was maintained in a sterile incubator at $37^{\circ} \mathrm{C}$ and $5 \% \mathrm{CO}_{2}$ for $48 \mathrm{~h}$.

\section{Frozen section and image}

Cartilage explants were washed in PBS three times and embedded in Optimal Cutting Temperature (OCT) compound (Tissue-Tek', SAKURAr, USA). Cartilage tissue was sliced into 4- $\mu \mathrm{m}$ sections (Thermo, CRYOSTAR NX50, Waltham, MA) and attached to microscope slides (Fisher).

The frozen section was washed in PBS three times. The Hoechst 33342 stock concentration was $1 \mathrm{mg} / \mathrm{mL}$, and the working concentration was $10 \mu \mathrm{g} / \mathrm{mL}$. The nuclei were stained with Hoechst 33342 for $5 \mathrm{~min}$ and then washed three times with PBS. The CAP-exosome/miR-140 penetration in cartilage explants was observed by a confocal laser scanning microscope (ZEISS, LSM 800, Germany).

\section{Results}

As shown in Figs. 2A and 2B, the exosome particle size show a peak value of $100 \mathrm{~nm}$ with no obvious heteropeaks. The results detected by transmission electron microscopy are shown in Fig. 2C. The exosomes purified by ultracentrifugation have a saucer-like structure with a double-layer membrane. The particle size ranges from 30 to $150 \mathrm{~nm}$, which is consistent with the morphological structure and particle size range of exosomes. As shown in Fig. 2D, the purified exosomes show high expressions of CD9, CD81, and flotillin and a low expression of calnexin.

Cy3-labeled miRNA-140 was loaded into CAP-exosomes or control exosomes by electroporation. By measuring the remaining free miR-140 in the solution and comparing it 
A
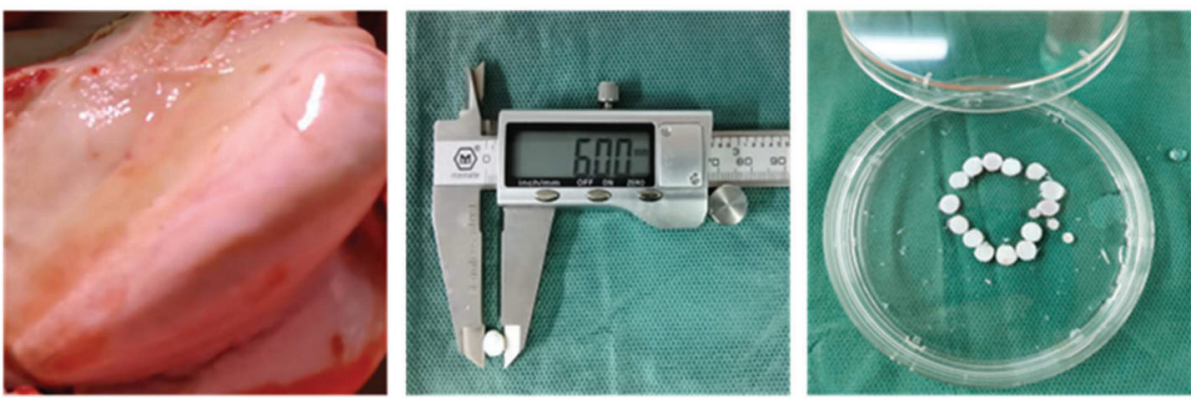

B
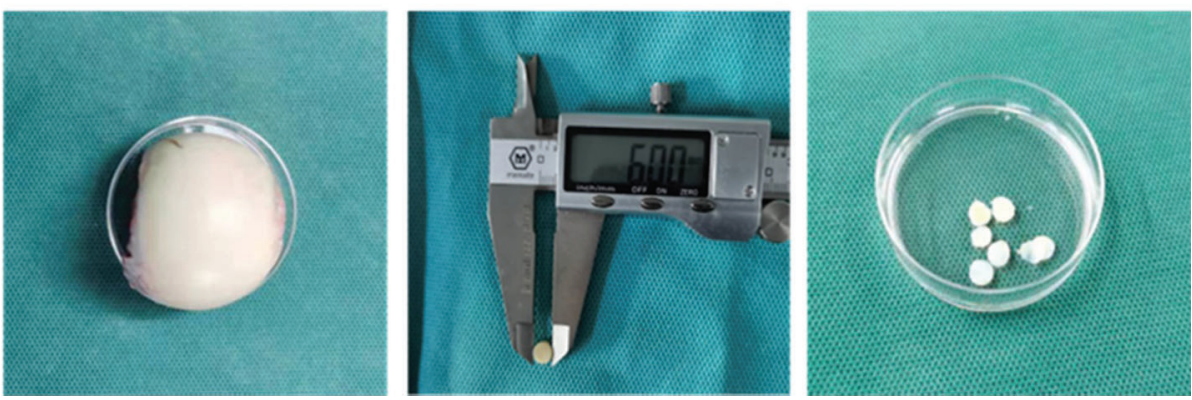

FIGURE 1. Schematic of the cartilage explant collection for the penetration assay. Cartilage explants were harvested and cultured. Fluorescent probe labelled exosomes were added to bovine cartilage explants (A) and human OA cartilage explants (B). After incubation, cartilage explants were washed tree times with $1 \times$ PBS, sections were mounted on glass slides and immediately observed under confocal microscope.
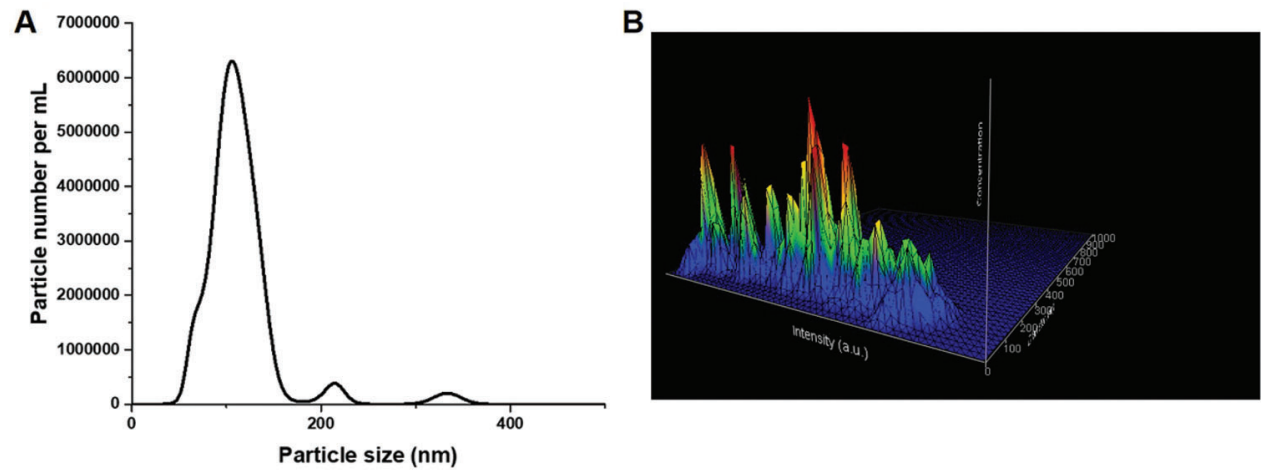

C
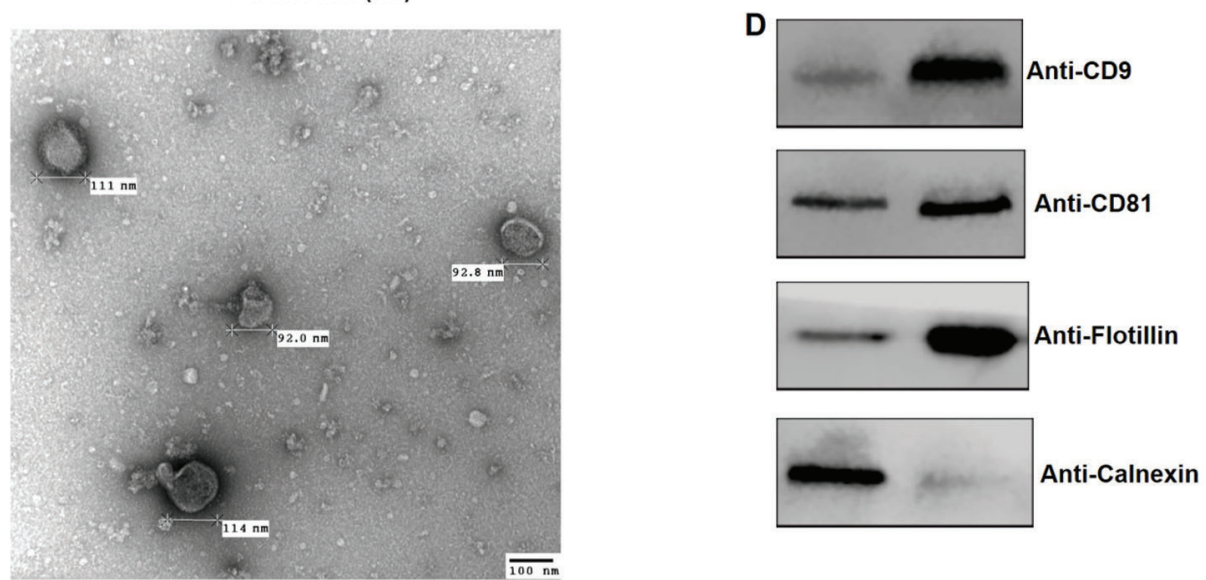

FIGURE 2. Characterization of exosomes. (A-B) Size distribution of the purified CAP-exosomes measured by NTA analysis. (C) Representative transmission electron microscopy images of CAP-exosomes. (D) Expression of the protein markers in CAP-exosomes by western blotting.

with the total input using a fluorometer (excitation at $550 \mathrm{~nm}$ and emission at $570 \mathrm{~nm}$ ), we calculated the loading efficiency of miRNA into exosome about $60 \%$. To track the penetration process by which exosomes deliver miR-140 in cartilage, we detected the Cy3 signal of CAP-exosome/miR-140 using frozen sections and took images using a confocal microscope. As shown in Fig. 3, miR-140 encapsulated in the CAP-exosomes (CAP-exosome/miR-140) was delivered 
A
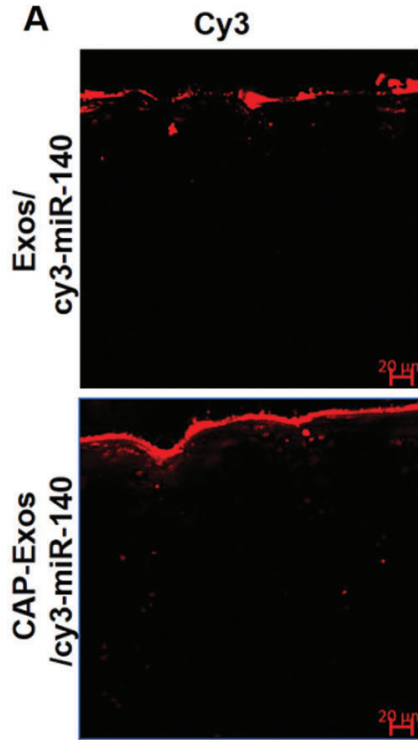

B
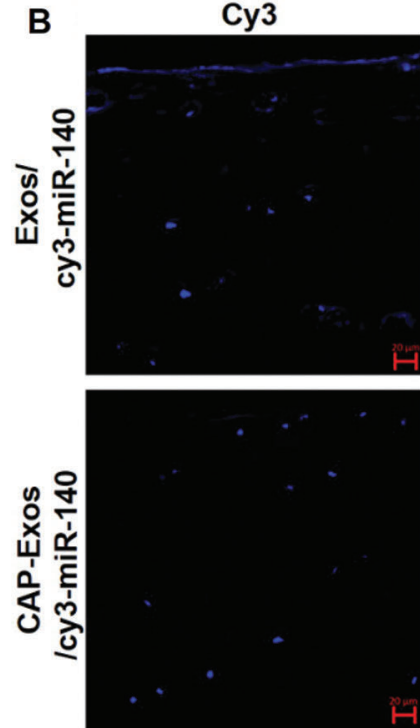

Hoechst
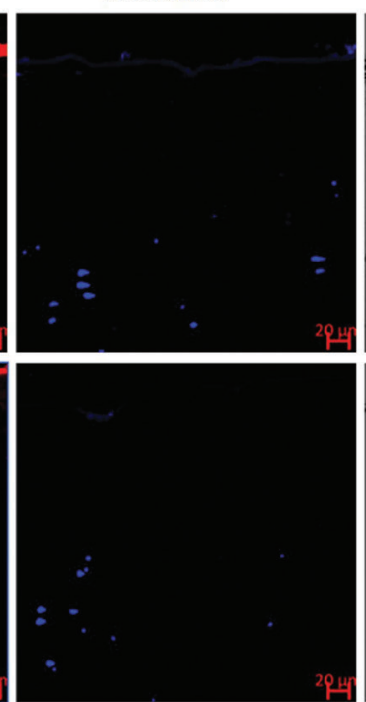

Hoechst
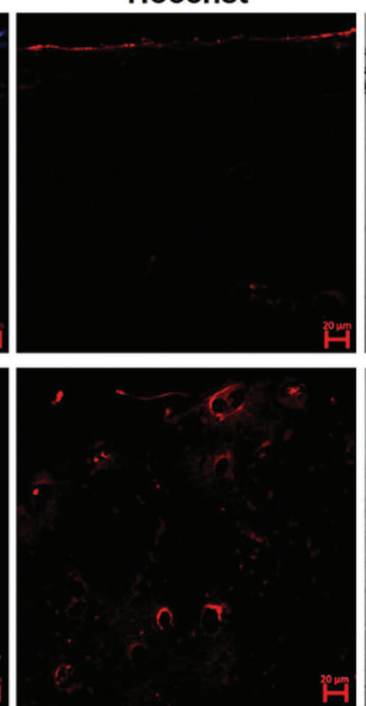

$\mathrm{BF}$
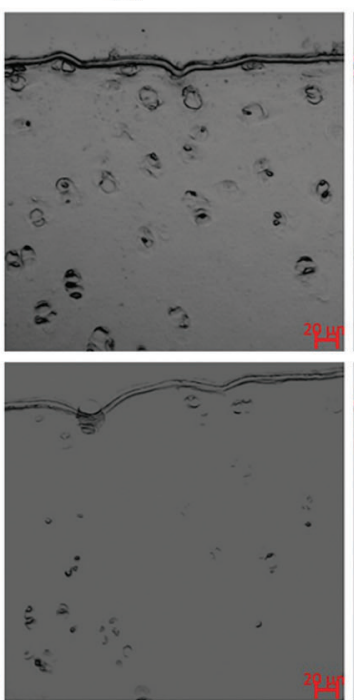

BF
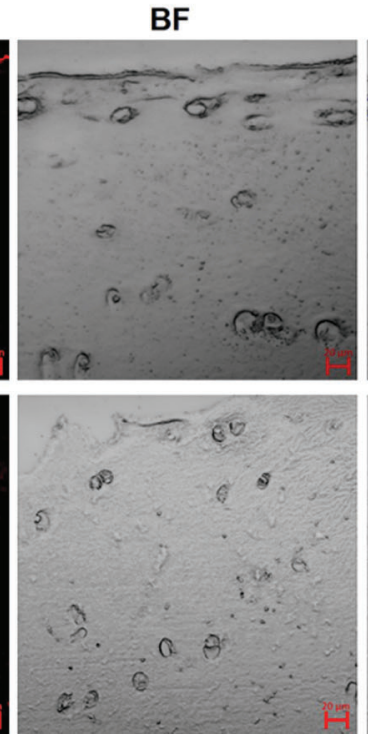
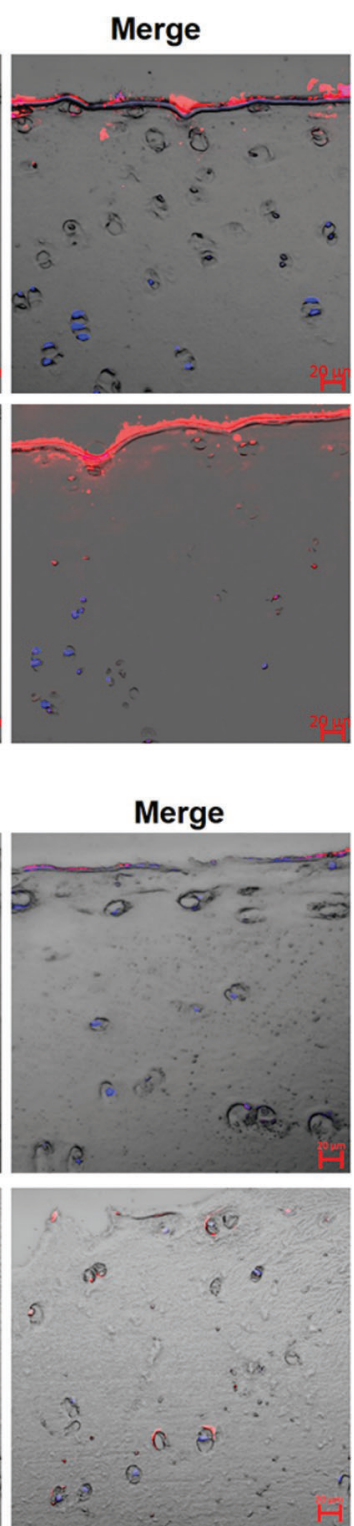

FIGURE 3. Chondrocyte targeted exosome penetration in the OA cartilage explants is deeper than no-targeted modified exosome. (A) Exosome penetration in the bovine cartilage explant. (B) Exosome penetration in the OA cartilage explant. The red fluorescence indicates the exosome. The blue fluorescence indicates the nucleus. Scale bar, $20 \mu \mathrm{m}$.

into the bovine cartilage explants (Fig. 3A) and human cartilage explants (Fig. 3B), while miR-140 delivered by the control exosomes was mainly enriched on the surface layer. Most of the CAP-exosome/miR-140 could penetrate bovine cartilage ex vivo, and the distribution of cy3-labeled miR-140 in human cartilage explants was more uniform and deeper.

\section{Discussion}

$\mathrm{OA}$ is an intractable disease since cartilage defects are challenging to manage. The greatest challenge for $\mathrm{OA}$ treatment comes from the inflammatory milieu of the joint cavity and the dense cartilage matrix with no blood supply. To address these obstacles, we established a cartilagetargeted nanocarrier delivery system. Dendritic cells (DCs) are popular cell sources for exosome preparation, as they can produce large quantities of exosomes with weak activation of $\mathrm{T}$ cells in vitro. DCs-derived exosomes have been considered as a promising alternative to DC-based vaccines (Pitt et al., 2016). In this system, the cartilage affinity peptide CAP was engineered on the DCs-derived exosome membrane. miR-140, a dual-factor that not only inhibits cartilage degradation but also promotes cartilage regeneration, was encapsulated into cartilage-target exosomes (Duan et al., 2020a; Liang et al., 2021a).

Extensive studies have demonstrated the role of exosomes in drug delivery (Duan et al., 2021a; Duan et al., 2021b; Duan et al., 2020b; Huang et al., 2021; Liang et al., 2021a; Xu et al., 2021). Additionally, the limitation of native exosomes in drug delivery has been recognized. Native exosomes may protect miR-140 from nuclease-induced degradation. However, without targeting ability, they will deliver miR-140 to many tissues or organs. Thus, it may severely compromise the treatment outcome. As miR-140 plays a vital role in the stability and maintenance of the cartilage matrix, it holds great potential as a therapeutic target for OA. 
To overcome another obstacle in OA treatment, we need a novel evaluation system for cartilage-targeted drug delivery. The most often used animal model for drug delivery is rodents, such as mice and rats. The mean thickness of mouse joint cartilage is $40-60 \mu \mathrm{m}$, and that of rats is $100 \mu \mathrm{m}$. However, the thickness of human knee cartilage is approximately 1-2 mm (Chang et al., 2016; Malda et al., 2013; Otterness et al., 2008). Equine and sheep models are also well established. They may be more relevant to further in vivo testing due to more similar cartilage thickness to human cartilage, more similar loading weights, and joint volume to humans. Different cartilage depths could result in discrepancies in treatment outcomes with the same therapeutics.

Mouse knee cartilage is very thin and not an appropriate model for studying cartilage penetration. In contrast, bovine cartilage is approximately $0.5-2.5 \mathrm{~mm}$ relative to that of the human knee. Both bovine joints and joints from OA patients with a knee joint replacement have been suggested to be ideal knee cartilage explants. For example, the bovine or human cartilage explant has been used to test the therapeutic effects of proteinase inhibitors, growth factors, and the matrix metalloproteinase (MMP) activities of OA (Chang et al., 2016; Fay et al., 2006; Homandberg et al., 2004; Jin et al., 2003; Reker et al., 2017; Wang et al., 2009). Moreover, cartilage explants extracted from OA patients have been used to detect the autologous leukocyte-poor platelet-rich plasma (LP-PRP) effects on inflammationassociated markers (Simental-Mendía et al., 2018). Compared with the chondrocyte model, the cells embedded in the explant model are located in the natural ECM, which is an ideal model for evaluating the penetration performance and effect of drugs. The source of human cartilage explants is limited, and their use is with ethical issues. However, the bovine cartilage explants are conveniently available. The bovine cartilage explants are recommended as practicable explant models to assess the functional properties of cartilage explants (Bian et al., 2010), chondroprotection (Kumar et al., 2019; Reker et al., 2017; Siebuhr et al., 2020), and cartilage penetration (Krishnan et al., 2018). For penetration assay, the bovine cartilage explant was used to detect the engineered sPLA2i loaded micelles that can penetrate deeply into the cartilage matrix and have good retention (Wei et al., 2015). Another study that used bovine cartilage explant found that amine-terminated polyamidoamine (PAMAM) dendritic polymer nanocarriers can improve cartilage tissue binding, penetration, and residence time. They can penetrate the body's thickness of bovine cartilage within 2 days. After IGF-1 protein was used for the treatment of arthritis, the residence time of IGF-1 protein in the knee joint of the rat was increased by 10 times (Geiger et al., 2018).

Our study results further demonstrated that cartilage explants from both bovine joints and patients with hip joint replacement are ideal models to test cartilage-targeted drug delivery as an initial in vitro system prior to further necessary in vivo studies. They can accurately monitor the process of therapeutic diffusion. In future studies, the explants could be further assayed by histochemistry analysis to define the nature of regenerated cartilage with no need for animal sacrifice.

\section{Conclusion}

The cartilage-targeted drug delivery system established in this study is a promising carrier to deliver therapeutics to cartilage defects. Ex vivo cartilage explants are an effective and economic system to evaluate the therapeutics in preclinical studies for OA treatment.

Availability of Data and Materials: All data generated or analyzed during this study are included in this published article.

Authors' Contribution: The authors confirm contribution to the paper as follows: study conception and design: Kan Ouyang, Yujie Liang, Li Duan; data collection: Kan Ouyang, Meiquan Xu, Xiao Xu, Caining Wen, Zhuan Qin, Yixin Xie; analysis and interpretation of results: Yujie Liang, Huawei Zhang; draft manuscript preparation: Kan Ouyang, Yujie Liang, Li Duan, Daping Wang. All authors reviewed the results and approved the final version of the manuscript.

Ethics Approval: This study has been approved by the Ethics Committees from Shenzhen Second People's Hospital (20201109001-FS01, date of approval: 12-23-2020).

Funding Statement: National Natural Science Foundation of China (Nos. 81972116, 81972085, 81772394, 31900046); Key Program of Natural Science Foundation of Guangdong Province (No. 2018B0303110003); Guangdong International Cooperation Project (No. 2021A0505030011); Shenzhen Science and Technology Projects (Nos. GJHZ20200731095606019, JCYJ20170817172023838, JCYJ20170306092215436, JCYJ20170413161649437); China Postdoctoral Science Foundation (No. 2020M682907); Special Funds for the Construction of High Level Hospitals in Guangdong Province.

Conflicts of Interest: The authors declare that they have no conflicts of interest to report regarding the present study.

\section{References}

Abramoff B, Caldera FE (2020). Osteoarthritis: Pathology, diagnosis, and treatment options. Medical Clinics 104: 293-311.

Armiento AR, Alini M, Stoddart MJ (2019). Articular fibrocartilageWhy does hyaline cartilage fail to repair? Advanced Drug Delivery Reviews 146: 289-305. DOI 10.1016/j.addr.2018.12.015.

Bian L, Stoker AM, Marberry KM, Ateshian GA, Cook JL, Hung CT (2010). Effects of dexamethasone on the functional properties of cartilage explants during long-term culture. The American Journal of Sports Medicine 38: 78-85. DOI 10.1177/ 0363546509354197.

Chang SH, Yasui T, Taketomi S, Matsumoto T, Kim-Kaneyama JR et al. (2016). Comparison of mouse and human ankles and establishment of mouse ankle osteoarthritis models by surgically-induced instability. Osteoarthritis Cartilage 24: 688-697. DOI 10.1016/j.joca.2015.11.008.

Duan L, Liang Y, Xu X, Xiao Y, Wang D (2020a). Recent progress on the role of miR-140 in cartilage matrix remodelling and its implications for osteoarthritis treatment. Arthritis Research \& Therapy 22: 194. DOI 10.1186/s13075-020-02290-0.

Duan L, Ouyang K, Wang J, Xu L, Xu X et al. (2021a). Exosomes as targeted delivery platform of CRISPR/Cas9 for therapeutic 
genome editing. ChemBioChem Early View 19: 164. DOI $10.1002 /$ cbic.202100359.

Duan L, Xu L, Xu X, Qin Z, Zhou X et al. (2021b). Exosome-mediated delivery of gene vectors for gene therapy. Nanoscale 13: 1387-1397. DOI 10.1039/D0NR07622H.

Duan L, Xu X, Xu L, Chen H, Li X et al. (2020b). Exosome-mediated drug delivery for cell-free therapy of osteoarthritis. Current Medicinal Chemistry 28: 6458-6483. DOI 10.2174/ 0929867327666201118161232.

El-Andaloussi S, Lee Y, Lakhal-Littleton S, Li J, Seow Y et al. (2012). Exosome-mediated delivery of siRNA in vitro and in vivo. Nature Protocols 7: 2112-2126. DOI 10.1038/nprot.2012.131.

Fay J, Varoga D, Wruck CJ, Kurz B, Goldring MB, Pufe T (2006). Reactive oxygen species induce expression of vascular endothelial growth factor in chondrocytes and human articular cartilage explants. Arthritis Research \& Therapy 8: R189. DOI 10.1186/ar2102.

Geiger BC, Wang S, Padera RF, Grodzinsky AJ, Hammond PT (2018). Cartilage-penetrating nanocarriers improve delivery and efficacy of growth factor treatment of osteoarthritis. Science Translational Medicine 10: 363. DOI 10.1126/ scitranslmed.aat 8800 .

Homandberg GA, Ummadi V, Kang H (2004). The role of insulinlike growth factor-I in hyaluronan mediated repair of cultured cartilage explants. Inflammation Research 53: 396404. DOI 10.1007/s00011-004-1276-y.

Huang J, Xiong J, Yang L, Zhang J, Sun S, Liang Y (2021). Cell-free exosome-laden scaffolds for tissue repair. Nanoscale 13: 8740-8750. DOI 10.1039/D1NR01314A.

Jin M, Emkey GR, Siparsky P, Trippel SB, Grodzinsky AJ (2003). Combined effects of dynamic tissue shear deformation and insulin-like growth factor I on chondrocyte biosynthesis in cartilage explants. Archives of Biochemistry and Biophysics 414: 223-231. DOI 10.1016/S0003-9861(03)00195-4.

Kleuskens MWA, Van Donkelaar CC, Kock LM, Janssen RPA, Ito K (2021). An ex vivo human osteochondral culture model. Journal of Orthopaedic Research 39: 871-879. DOI 10.1002/ jor.24789.

Kou L, Xiao S, Sun R, Bao S, Yao Q, Chen R (2019). Biomaterialengineered intra-articular drug delivery systems for osteoarthritis therapy. Drug Delivery 26: 870-885. DOI 10.1080/10717544.2019.1660434.

Krishnan Y, Rees HA, Rossitto CP, Kim SE, Hung HHK et al. (2018). Green fluorescent proteins engineered for cartilage-targeted drug delivery: Insights for transport into highly charged avascular tissues. Biomaterials 183: 218-233. DOI 10.1016/ j.biomaterials.2018.08.050.

Kumar S, Adjei IM, Brown SB, Liseth O, Sharma B (2019). Manganese dioxide nanoparticles protect cartilage from inflammation-induced oxidative stress. Biomaterials 224: 119467. DOI 10.1016/j.biomaterials.2019.119467.

Liang Y, Duan L, Lu J, Xia J (2021a). Engineering exosomes for targeted drug delivery. Theranostics 11: 3183-3195. DOI 10.7150/thno.52570.

Liang Y, Xu X, Li X, Xiong J, Li B et al. (2020). Chondrocyte-targeted microRNA delivery by engineered exosomes toward a cellfree osteoarthritis therapy. ACS Applied Materials \& Interfaces 12: 36938-36947. DOI 10.1021/acsami.0c10458.

Liang Y, Xu X, Xu L, Prasadam I, Duan L et al. (2021b). Non-surgical osteoarthritis therapy, intra-articular drug delivery towards clinical applications. Journal of Drug Targeting 29: 609616. DOI 10.1080/1061186X.2020.1870231.

Malda J, de Grauw JC, Benders KE, Kik MJ, van de Lest CH et al. (2013). Of mice, men and elephants: The relation between articular cartilage thickness and body mass. PLoS One 8: e57683. DOI 10.1371/journal.pone.0057683.

Maudens P, Jordan O, Allémann E (2018). Recent advances in intraarticular drug delivery systems for osteoarthritis therapy. Drug Discovery Today 23: 1761-1775. DOI 10.1016/j. drudis.2018.05.023.

Nganvongpanit K, Itthiarbha A, Ong-Chai S, Kongtawelert P (2008). Evaluation of serum chondroitin sulfate and hyaluronan: Biomarkers for osteoarthritis in canine hip dysplasia. Journal of Veterinary Science 9: 317-325. DOI 10.4142/ jvs.2008.9.3.317.

Nganvongpanit K, Pothacharoen P, Chaochird P, Klunklin K, Warrit $\mathrm{K}$ et al. (2009). Prospective evaluation of serum biomarker levels and cartilage repair by autologous chondrocyte transplantation and subchondral drilling in a canine model. Arthritis Research \& Therapy 11: R78. DOI 10.1186/ar2709.

Otterness IG, Le Graverand MP, Eckstein F (2008). Allometric relationships between knee cartilage volume, thickness, surface area and body dimensions. Osteoarthritis Cartilage 16: 34-40. DOI 10.1016/j.joca.2007.05.010.

Pitt JM, André F, Amigorena S, Soria JC, Eggermont A et al. (2016). Dendritic cell-derived exosomes for cancer therapy. The Journal of Clinical Investigation 126: 1224-1232. DOI 10.1172/JCI81137.

Reker D, Kjelgaard-Petersen CF, Siebuhr AS, Michaelis M, Gigout A et al. (2017). Sprifermin (rhFGF18) modulates extracellular matrix turnover in cartilage explants ex vivo. Journal of Translational Medicine 15: 250. DOI 10.1186/s12967-017-1356-8.

Siebuhr AS, Werkmann D, Bay-Jensen AC, Thudium CS, Karsdal MA et al. (2020). The anti-ADAMTS-5 Nanobody( $\left({ }^{\circ}\right)$ M6495 protects cartilage degradation ex vivo. International Journal of Molecular Sciences 21: 5992. DOI 10.3390/ijms21175992.

Simental-Mendía M, Vilchez-Cavazos F, García-Garza R, Lara-Arias J, Montes-De-Oca-Luna R et al. (2018). The matrix synthesis and anti-inflammatory effect of autologous leukocyte-poor platelet rich plasma in human cartilage explants. Histology and Histopathology 33: 609-618.

Wang B, Chen P, Jensen AC, Karsdal MA, Madsen SH et al. (2009). Suppression of MMP activity in bovine cartilage explants cultures has little if any effect on the release of aggrecanasederived aggrecan fragments. BMC Research Notes 2: 259. DOI 10.1186/1756-0500-2-259.

Wei B, Zong M, Yan C, Mao F, Guo Y et al. (2015). Use of quantitative MRI for the detection of progressive cartilage degeneration in a mini-pig model of osteoarthritis caused by anterior cruciate ligament transection. Journal of Magnetic Resonance Imaging 42: 1032-1038. DOI 10.1002/ jmri.24862.

Wuelling M, Vortkamp A (2014). Cartilage explant cultures. In: Hilton M (ed.), Skeletal Development and Repair. Methods in Molecular Biology. Totowa, NJ: Humana Press.

Xu X, Liang Y, Li X, Ouyang K, Wang M et al. (2021). Exosomemediated delivery of kartogenin for chondrogenesis of synovial fluid-derived mesenchymal stem cells and cartilage regeneration. Biomaterials 269: 120539. DOI 10.1016/j. biomaterials.2020.120539. 